\title{
X-linked intellectual disability, Brooks type
}

INSERM

\section{Source}

INSERM. (1999). Orphanet: an online rare disease and orphan drug data base. $\underline{X \text {-linked }}$ intellectual disability, Brooks type. ORPHA:3056

X-linked intellectual disability, Brooks type is a rare X-linked intellectual disability syndrome characterized by failure to thrive, speech delay, intellectual disability, muscle hypotonia, spastic diplegia, optic atrophy with myopia, and distinct facial features (including triangular face, bifrontal narrowness, deeply set eyes, low-set/cupped ears, prominent nose, short philtrum, and thin upper lip with tented morphology) that can be evident from birth. Additional manifestations reported in some patients include large joint contractures and pectus excavatum (which become more evident with age) and seizures. 